\title{
Convex fung-type potentials for biological tissues
}

\author{
Salvatore Federico • Alfio Grillo • \\ Gaetano Giaquinta • Walter Herzog
}

Published online: 11 December 2007

(C) Springer Science+Business Media B.V. 2007

\section{Erratum to: Meccanica \\ DOI 10.1007/s11012-007-9090-6}

In the final stage of the publication process of the above-mentioned article, the following author corrections were inadvertently not incorporated in the final version;

Page 3, left column, last line

"... four-linear form on..."

should read

"... four-linear application on..."
Page 3, right column, fourth line

"... bilinear form on..."

should read

"... bilinear application on..."

Page 4, right column, eighth line

"A full symmetric tensor..."

should read

"A full symmetric isotropic tensor..."

The online version of the original article can be found under doi: 10.1007/s11012-007-9090-6.

S. Federico $\cdot$ W. Herzog $(\bowtie)$

Human Performance Laboratory, Faculty of Kinesiology, The University of Calgary, 2500 University Drive NW,

Calgary, AB, T2N 1N4, Canada

e-mail: walter@kin.ucalgary.ca

A. Grillo · G. Giaquinta

Dipartimento di Metodologie Fisiche e Chimiche per

l'Ingegneria, Facoltà di Ingegneria, Università di Catania,

Viale Andrea Doria 6, 98125 Catania, Italy

A. Grillo · G. Giaquinta

Consorzio Nazionale Interuniversitario per le Scienze

Fisiche della Materia, Sezione di Catania, Viale Andrea

Doria 6, 98125 Catania, Italy 